\title{
ВИКОРИСТАННЯ ТЕХНОЛОГІЇ DАТА MINING ІЗ МЕТОЮ ДИФЕРЕНЦІАЛЬНОЇ ДІАГНОСТИКИ КОМОРБІДНИХ СТАНІВ ХРОНІЧНОГО ПАНКРЕАТИТУ Й АСКАРИДОЗУ НА ПІДСТАВІ ДАНИХ КЛІНІЧНОЇ СИМПТОМАТИКИ Й УЛЬТРАЗВУКОВИХ ДОСЛІДЖЕНЬ
}

\author{
В. П. Марценюк, Л. С. Бабінець ${ }^{1}$, Ю. В. Дроняк ${ }^{1}$ \\ Університет Бельсько-Бяла, Польща \\ ${ }^{1}$ ДВНЗ «Тернопільський державний медичний університет \\ імені І. Я. Горбачевського МОЗ України»
}

Для діагностування коморбідності хронічного панкреатиту й аскаридозу використана методика побудови дерева рішень, що ґрунтується на алгоритмі С5.0. При цьому можуть бути використані дані як клінічної симптоматики, так і ультразвукового дослідження. Для кожного з видів досліджень, а також для їх сукупності побудовано окреме дерево рішень. Досліджено похибка алгоритму.

Ключові слова: хронічний панкреатит, аскаридоз, коморбідність, клінічна симптоматика, УзД, дерево рішень, data mining, $\mathrm{R}, \mathrm{C} 5.0$.

\section{AN APPLICATION OF DATA MINING TECHNOLOGY WITH AIM OF DIFFERENTIAL DIAGNOSTICS OF THE COMORBIDIC STATES OF CHRONIC PANCREATITIS AND ASCARIDOSIS ON THE BASIS OF DATA OF CLINICAL SYMPTOMATOLOGY AND ULTRASONIC RESEARCHES}

V. P. Martsenyuk, L. S. Babinets ${ }^{1}$, Yu. V. Dronyak ${ }^{1}$ University of Bielsko-Biala, Poland

${ }^{1}$ SHEI "I. Ya. Gorbachevsky Ternopil state medical university of MH of Ukraine"

\begin{abstract}
For diagnostics of chronic pancreatitis and ascaridosis comorbidity methodology of decision tree construction based on C5.0 algorithm is used. Data of both clinical symptomatology and ultrasonography can be applied. For each of types of researches and also for their totality a separate decision tree is built. The error of algorithm is investigated.
\end{abstract}

Key words: chronic pancreatitis, ascaridosis, comorbidity, clinical symptomatology, ultrasonic, decision tree, data mining, R, C5.0.

\section{ИСПОЛЬЗОВАНИЕ ТЕХНОЛОГИИ DАТА MINING С ЦЕЛЬЮ ДИФФЕРЕНЦИАЛЬНОЙ ДИАГНОСТИКИ КОМОРБИДНЫХ СОСТОЯНИЙ ХРОНИЧЕСКОГО ПАНКРЕАТИТА И АСКАРИДОЗА НА ОСНОВАНИИ ДАННЫХ КЛИНИЧЕСКОЙ СИМПТОМАТИКИ И УЛЬТРАЗВУКОВЫХ ИССЛЕДОВАНИЙ}

\author{
В. П. Марценюк, Л. С. Бабинец ${ }^{1}$, Ю. В. Дроняк ${ }^{1}$ \\ Университет Бельско-Бяла, Польша

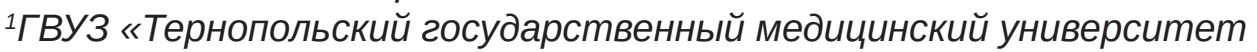 \\ имени И. Я. Горбачевского МЗ Украины»
}

\begin{abstract}
Для диагностирования коморбидности хронического панкреатита и аскаридоза использована методика построения дерева решений, основанная на алгоритме С5.0. При этом могут быть использованы данные, как клинической симптоматики, так и ультразвукового исследования. Для каждого из видов исследований, а также для их совокупности построено отдельное дерево решений. Исследована погрешность алгоритма.
\end{abstract}

Ключевые слова: хронический панкреатит, аскаридоз, коморбидность, клиническая симптоматика, УзИ, дерево решений, data mining, R, C5.0.

(c) В. П. Марценюк, Л. С. Бабінець, Ю. В. Дроняк 
Введение. Распространенность заболеваний поджелудочной железы (ПЖ) и их место в общей структуре болезней органов желудочно-кишечного тракта сложно оценить. На сегодня распространенность хронического панкреатита (ХП) среди населения Украины, по некоторым данным, составляет 25-30 человек на 100 тыс. населения. За последние 30-40 лет количество больных ХП увеличилось вдвое, первичная инвалидизация достигла $15 \%$ [3, 7].

Течение ХП в большинстве случаев не является изолированным, растет количество лиц с сочетанной патологией, а поражения органов пищеварения отягощают и усложняют течение других заболеваний. Исследования ряда авторов свидетельствуют о том, что в 70-90 \% случаев заболевания желудочно-кишечного тракта сочетаются между собой или сопровождаются заболеваниями других органов и систем [6]. В последние годы все большее внимание исследователей уделяется изучению сочетания гастроэнтерологических заболеваний с глистной инвазией. Это, в свою очередь, определяет необходимость углубления знаний о патогенетических звеньях при сочетанной патологии и разработок эффективных лечебных и профилактических мероприятий.

Коморбидность ХП и аскаридоза является достаточно актуальной проблемой в настоящее время. ХП — частый спутник гельминтозов, особенно аскаридоза.

Клиническая симптоматика ХП чрезвычайно богата и полиморфна в своих проявлениях, однако малоспецифична [2, 3, 6]. Классически можно выделить основные критерии клинического течения ХП посиндромно. Клинически ХП характеризуется двумя ведущими синдромами: рецидивирующей и/или постоянной абдоминальной болью и/или экзокринной недостаточностью ПЖ [6]. У части пациентов боль не связана с приемом пищи, у примерно 15 \% больных ХП боль отсутствует [7]. При длительном течении ХП, когда отмечается прогрессирование диффузного фиброза в ткани ПЖ, возникает и постепенно углубляется трофологическая недостаточность (ТН), характеризующаяся синдромами дефицита энергии, белка, витаминов, микроэлементов и электролитов [2, 3]. На фоне прогрессирующей функциональной недостаточности ПЖ и углубления ТН исчерпываются запасы нутриентов в тканях, что сначала ведет к биохимическим и функциональным изменениям, а в дальнейшем проявляется многочисленными и сложными клиническими симптомами $[6,7]$.
Аскаридоз характеризуется признаками хронической интоксикации и аллергизации человеческого организма, нарушением функционирования иммунной системы, органов дыхания и пищеварительного тракта, миалгией, лимфаденопатией, железодефицитной анемией. Как результат, происходит снижение показателей здоровья, а следовательно, и качества жизни пациентов.

Наличие в организме человека аскарид маскируется такими характерными для многих заболеваний проявлениями, как хроническая усталость, изменение аппетита (чаще его ухудшение), нервозность, нарушение моторики желудочно-кишечного тракта (тошнота, иногда рвота, запор, диарея, метеоризм), слюнотечение, анемия и другие изменения в крови - повышение СОЭ, эозинофилия; отмечается боль в различных группах мышц, суставах, головная боль, «беспричинное» периодическое повышение температуры тела до субфебрильных значений (37-38 $\left.{ }^{\circ} \mathrm{C}\right)$, бессонница и другие нарушения сна, гиповитаминозы, различные кожные проявления, аллергические признаки, частые заболевания дыхательной системы (иногда вплоть до бронхиальной астмы), бруксизм (скрежет зубами во время сна) [8].

Из всех органов, расположенных в брюшной полости человека, ПЖ наименее доступна для обследования, поскольку она располагается сзади и книзу желудка, за петлями тонкой кишки и за поперечно-ободочной кишкой, поэтому пропальпировать ее можно только в том случае, когда орган значительно увеличен и изменен [5]. Но при такой клинической ситуации у больного в подавляющем большинстве случаев проявляется яркая развернутая клиническая картина заболевания, и сомнений в том, что патологический процесс задевает указанный орган, у квалифицированного врача практически не возникает.

Именно поэтому ультразвуковое исследование (УЗИ) ПЖ является одним из наиболее достоверных и доступных в повседневной клинической практике инструментальных исследований, своевременное выполнение которого помогает визуализировать орган в самых разных проекциях и оценить его состояние практически в любой момент времени. Чувствительность данного исследования в случае диагностики ХП достигает в среднем 70,0-80,0 \%, а специфичность - 80,090,0 \%. При этом в 10,0-20,0 \% случаев - при подтвержденном ХП $[1,4]$. 
Основными эхографическими признаками ХП являются:

- неоднородность эхоструктуры ПЖ преимущественно за счет участков повышенной эхогенности;

- неравномерное расширение главного панкреатического протока;

_ - кальцификаты в паренхиме и камни в панкреатическом протоке;

— изменение размеров ПЖ (увеличение при обострении из-за отека или уменьшение в результате атрофии и фиброза на поздних стадиях заболевания);

- неравномерное повышение эхогенности ПЖ;

- неровные, нечеткие контуры ПЖ;

- боль при нажатии датчиком или пальпации ПЖ под контролем УЗИ;

- дополнительные эхографические признаки ХП: перегиб больших сосудов, холедоха, наличие ретенционных кист и псевдокист;

- отсутствие изменений ПЖ на ранних стадиях ХП при проведении обычного трансабдоминального УЗИ.

Цель работы: показать возможности метода индукции дерева решений для программной реализации в системе принятия решений относительно коморбидности ХП и аскаридоза.

Материалы и методы исследования. Обследовано 90 пациентов с ХП в возрасте от 18 до 77 лет (средний возраст 50,5 \pm 13,9 года), из них 56 (62,2 \%) женщин и 34 (37,7 \%) мужчины. Пациенты были распределены на две группы: 1-я - 60 пациентов с ХП в сочетании с аскаридозом, 2-я - 30 пациентов с изолированным ХП. Всем пациентам с ХП было проведено общеклиническое обследование и выполнены УзИ ПЖ и лабораторные исследования.

Результаты и их обсуждение. Применение алгоритма индукции дерева решений. Математически задача индукции дерева решений формулируется следующим образом. Имеем множество $D$, которое содержит $N$ наборов учебных данных. При этом каждый набор $\left(A_{1}^{i}, A_{2}^{i}, \ldots, A_{p}^{i}, C^{i}\right)$ состоит из исходных данных - атрибутов $A_{1}, \ldots, A_{p}$ и выходящих данных - атрибутов класса $C$. Атрибуты $A_{1}, \ldots, A_{p}$ могут иметь как числовые, так и категориальные значения. Атрибут класса $\boldsymbol{C}$ принимает одно из К дискретных значений: $\boldsymbol{C} \in\{\mathbf{1}, \ldots, \mathbf{K}\}$. Целью является прогнозирование деревом решений значений атрибута класса $\boldsymbol{C}$ на основании значений атрибутов $A_{1}, \ldots, A_{p}$. При этом следует максимизировать точность прогнозирования атрибута класса $\mathrm{C}$, а именно $P\{C=c\}$ на терминальных узлах для свободного $C \in\{1, \ldots, K\}$. Алгоритмы индукции дерева решений автоматически разбивают на узлы значений числовых атрибутов $A_{i}$ на два интервала: $A_{i} \leq x_{i}$ и $A_{i}>x_{i}$, а категориальные атрибуты $A_{j}$ - на два подмножества: $A_{j} \in S_{j}, A_{j} \notin S_{j}$. Разбиение численных атрибутов основывается, как правило, на принципе энтропии или индексе Джини (Марценюк В. П., 2015). Процесс разбиения рекурсивно повторяется до тех пор, пока не будет наблюдаться улучшение точности прогнозирования. Последний шаг включает исключение узлов для избегания оверфитинга модели. В результате должны получить множество правил, которые идут от корня к каждому терминальному узлу, содержат неравенства для численных атрибутов и условия включения для категориальных атрибутов.

Метод индукции дерева решений. За основу взято следующую рекурсивную процедуру работы (Марценюк В. П., 2015).

\section{Генерация дерева решений.}

Исходные данные: $D$ - множество учебных наборов данных $\left(A_{1}^{i}, A_{2}^{i}, \ldots, A_{p}^{i}, C^{i}\right)$.

Выходящие данные: дерево решений.

\section{Метод:}

1. Создать узел $N$.

2. Если все наборы в $D$ принадлежат к общему классу $C$, тогда вернуть узел $N$ как листок с названием класса $C$.

3. Если список атрибутов (а значит и $D$ ) является пустым, тогда вернуть узел $N$ как листок с названием самого распространённого класса в $D$.

4. Применить Алгоритм отбора атрибута из списка атрибутов и для множества $D$ с целью отыскания «наилучшего» атрибута деления.

5. Удалить атрибут деления из списка атрибутов.

6. Для каждого условия деления $J$ для атрибута деления рассмотрим $D_{j}-$ множество наборов из $D$, которые удовлетворяют условию деления $J$.

7. Если $D_{j}$ - пустое, тогда присоединить к узлу $N$ лист под заглавием самого распространённого класса в $D_{j}$, иначе - присоединить к $N$ узел, который возвращается рекурсивным вызовом метода Генерация дерева решений с исходными данными $D_{j}$ и список атрибутов.

8. Конец цикла шага 6.

9. Вернуть узел $N$. 
В основу Алгоритма отбора атрибута на $\boldsymbol{J}$-м шаге рекурсии положен следующий информационный показатель:

$\operatorname{Gain}\left(A_{i}\right)=\operatorname{Info}\left(D_{j}\right)-\operatorname{Info}{ }_{A_{i}}\left(D_{j}\right)$.

Здесь

$$
\operatorname{Info}\left(D_{j}\right)=-\sum_{k=1}^{K} p_{k}^{j} \log _{2}\left(p_{k}^{j}\right)
$$

информация, необходимая для классификации набора $A_{1}, \ldots, A_{p} \mathbf{в} D_{j}$,

$$
\operatorname{Info}_{A_{i}}\left(D_{j}\right)=\sum_{l=1}^{K_{i}} \frac{\#\left(D_{j}^{l}\right)}{\#\left(D_{j}\right)} \operatorname{Info}\left(D_{l}\right)(3)
$$

информация, необходимая для классификации $A_{1}, \ldots, A_{p}$ в $D_{j}$ после деления $D_{j}$ на подмножества $D_{j}^{l}$ соответственно к значениям атрибута $A_{i}$.

В формуле (2) вероятность того, что любой набор из $D_{j}$ принадлежит множеству $C_{k D_{j}}$ оценивается как

$$
p_{k}^{j}=\frac{\#\left(C_{k, D_{j}}\right)}{\#\left(D_{j}\right)},
$$

где $C_{k D_{j}}$ - множество наборов из $D_{j}$, для которых атрибут класса $C=k$. Здесь $\#(\bullet)-$ количество элементов в множестве.

В формуле (3) $\frac{\#\left(D_{j}^{l}\right)}{\#\left(D_{j}\right)}-$ оценка вероятности того, что любой набор из $D_{j}$ принадлежит множеству $D_{j}^{l}$, где $D_{j}^{l}-$ множество наборов из $D_{j}$, для которых атрибут $A_{i}=a_{i}^{l}$. Здесь атрибут $A_{i} \in\left\{a_{i}^{1}, a_{i}^{2}, \ldots, a_{i}^{K_{i}}\right\}$.

Таким образом, Gain( $\left.A_{i}\right)$ оценивает уменьшение информации, необходимой для классификации любого набора данных в $D_{j}$ за счёт известного значения атрибута $A_{i}$. Из наличных атрибутов на каждом узле дерева решений для условия деления следует выбирать атрибут $A_{i^{*}}$ с наибольшим значением $\operatorname{Gain}\left(A_{i^{*}}\right)$. В результате такого выбора для завершения процесса классификации набора данных в $D_{j}$ будет требоваться как можно меньше информации.

При применении вышеописанного алгоритма для построения дерева решений использованы такие наборы атрибутов (табл. 1, 2). При построении дерева с учётом данных как клинической симптоматики, так и УЗИ, использована комбинация атрибутов таблиц 1 и 2.

В работе использована реализация алгоритма на языке R с помощью пакета C5.0. Построенные деревья решений представлены на рис. 1-3.
Таблица 1

\section{Атрибуты для индукции дерева решений на основании клинической симптоматики}

\begin{tabular}{|c|c|c|}
\hline $\begin{array}{c}\text { Обозначение } \\
\text { атрибута }\end{array}$ & $\begin{array}{c}\text { Описание } \\
\text { атрибута }\end{array}$ & $\begin{array}{c}\text { Тип значений } \\
\text { атрибута } \\
\text { фактор }\end{array}$ \\
\hline A1 & Пол & целочисленный \\
\hline A3 & Боль в животе & фактор \\
\hline A4 & Тошнота & фактор \\
\hline A5 & Рвота & фактор \\
\hline A6 & Изжога & фактор \\
\hline A7 & Горечь во рту & фактор \\
\hline A8 & Отрыжка & фактор \\
\hline A9 & Вздутие & фактор \\
\hline А10 & Запор & фактор \\
\hline A11 & Диарея & фактор \\
\hline А12 & $\begin{array}{c}\text { Чередование за- } \\
\text { пора и диареи }\end{array}$ & фактор \\
\hline А13 & $\begin{array}{c}\text { Хороший аппе- } \\
\text { тит }\end{array}$ & фактор \\
\hline A14 & $\begin{array}{c}\text { Тяжесть в } \\
\text { эпигастрии }\end{array}$ & фактор \\
\hline А15 & $\begin{array}{c}\text { Ощущение пере- } \\
\text { полнения }\end{array}$ & фактор \\
\hline А16 & Общая слабость & фактор \\
\hline А17 & $\begin{array}{c}\text { Длительность } \\
\text { заболевания }\end{array}$ & целочисленный \\
\hline
\end{tabular}

Анализ полученных результатов. Анализируя дерево решений, построенное на основании данных клинической симптоматики (рис. 1), установили, что по информативности на первом месте находится наличие боли в животе. При ее отсутствии алгоритм предлагает вопрос о диарее. При ее наличии имеем с вероятностью $\mathrm{P}=1$ ХП (5 случаев). При отсутствии диареи система предлагает вопрос об ощущении горечи во рту. При ее наличии имеем с вероятностью $\mathrm{P}=1$ ХП (2 случая). При отсутствии уточняем пол. Для женщин с вероятностью $\mathrm{P}=1$ (4 случая) имеем коморбидность ХП с аскаридозом. Для мужчин уточняем возраст. Для мужчин моложе 45 лет с вероятностью $\mathrm{P}=1$ (4 случая) имеем коморбидность ХП с аскаридозом, для мужчин старше 45 лет - с вероятностью $\mathrm{P}=0,75$ (3 случая) отсутствие аскаридоза, с вероятностью $\mathrm{P}=0,25$ (1 случай) - его наличие. 
Таблица 2

\section{Атрибуты для индукции дерева решений на основании анализа данных УЗИ}

\begin{tabular}{|c|c|c|}
\hline $\begin{array}{c}\text { Обозначение } \\
\text { атрибута }\end{array}$ & $\begin{array}{c}\text { Описание } \\
\text { атрибута }\end{array}$ & $\begin{array}{c}\text { Тип значений } \\
\text { атрибута }\end{array}$ \\
\hline A18 & $\begin{array}{c}\text { Размер головки } \\
(11-30 \text { мм) }\end{array}$ & фактор \\
\hline A19 & $\begin{array}{c}\text { Размер тела } \\
\text { (8-21 мм) }\end{array}$ & фактор \\
\hline A20 & $\begin{array}{c}\text { Размер хвоста } \\
\text { (15-20 мм) }\end{array}$ & фактор \\
\hline A21 & Утолщенная & фактор \\
\hline A22 & Уплотненная & фактор \\
\hline A23 & $\begin{array}{l}\text { Диффузные } \\
\text { изменения }\end{array}$ & фактор \\
\hline A24 & $\begin{array}{c}\text { Холмистые } \\
\text { контуры }\end{array}$ & фактор \\
\hline A25 & $\begin{array}{c}\text { Нечеткие, } \\
\text { неровные } \\
\text { контуры } \\
\text { (размытые) } \\
\end{array}$ & фактор \\
\hline A26 & Гидрофильная & фактор \\
\hline A27 & $\begin{array}{c}\text { Фиброзные изме- } \\
\text { нения } \\
\end{array}$ & фактор \\
\hline A28 & $\begin{array}{c}\text { Эхогенность } \\
\text { повышенная, по- } \\
\text { ниженная } \\
\end{array}$ & фактор \\
\hline A29 & $\begin{array}{c}\text { Кальцинаты в } \\
\text { железе, протоках }\end{array}$ & фактор \\
\hline A30 & $\begin{array}{c}\text { Эхоструктура } \\
\text { неоднородная } \\
\text { (мелкозернистая) }\end{array}$ & фактор \\
\hline
\end{tabular}

При наличии абдоминальной боли встает вопрос об общей слабости. При ее наличии встает вопрос о тошноте. При наличии тошноты с вероятностью $\mathrm{P}=0,96$ (27 случаев из 28) присутствует аскаридоз. При отсутствии тошноты уточняем данные об аппетите. Если аппетит плохой, то с вероятностью $\mathrm{P}=0,9$ (17 из 19) имеем аскаридоз. Если аппетит хороший, то уточняем возраст. Если пациент моложе 41 года (включительно), то с вероятностью $\mathrm{P}=1$ аскаридоза нет (5 случаев). Если старше, то уточняем длительность болезни. Если болезнь длится меньше либо равна 10 годам, то с вероятностью $\mathrm{P}=1$ (7 случаев) имеем наличие аскаридоза. Если больше 10 лет, то с вероятностью $\mathrm{P}=0,7$ (2 случая из 3 ) аскаридоза нет. И наоборот.

При отсутствии общей слабости имеем соответствующее поддерево. Первым уточняем вопрос об ощущении тяжести в правом/левом подреберье (эпигастрии). При ее наличии имеем с вероятностью $\mathrm{P}=0,8$ аскаридоз. При ее отсутствии спрашиваем о вздутии. Если вздутия нет, то уточняем длительность болезни. Если длительность меньше 9 лет, то с вероятностью $\mathrm{P}=1$ аскаридоза нет. Если больше, то с вероятностью $\mathrm{P}=0,7$ аскаридоз есть (2 случая из 3). Если вздутие есть, то встает вопрос об изжоге. Если изжоги нет, то с вероятностью $\mathrm{P}=0,8$ имеем аскаридоз (4 случая из 5). Если изжога есть, то с вероятностью $\mathrm{P}=1$ аскаридоза не имеется.

Анализируя дерево решений, построенное на основании данных УЗИ (рис. 2), выявили, что на первом месте по информативности для диагностики коморбидности стоит увеличение размеров головки ПЖ (11-30 мм). При ее увеличении с вероятностью $\mathrm{P}=1$ имеем коморбидность ХП и аскаридоза (в нашем исследовании таким образом она определена у 10 пациентов). При нормальных размерах головки ПЖ следующим по информативности является наличие нечеткого неровного размытого контура. При его наличии вероятность коморбидного аскаридоза $-\mathrm{P}=0,2$ (11 пациентов), вероятность его отсутствия - $\mathrm{P}=0,8$ (39 пациентов). При отсутствии нечеткого неровного размытого контура алгоритм предлагает анализировать эхоструктуру. При отсутствии неоднородной (мелкозернистой) эхоструктуры с вероятностью $\mathrm{P}=0,6$ аскаридоз отсутствует (11 пациентов) и с вероятностью $\mathrm{P}=0,4-$ присутствует (7 пациентов). При наличии мелкозернистой эхоструктуры для дальнейшей диагностики необходимо исследовать диффузные изменения в ПЖ. При их наличии имеем с вероятностью $\mathrm{P}=0,8$ наличие аскаридоза (10 пациентов), с вероятностью $\mathrm{P}=0,2$ его отсутствие (2 пациента). При отсутствии диффузных изменений в ПЖ последним следует проанализировать наличие холмистых контуров. При их отсутствии имеем с вероятностью $\mathrm{P}=0,2$ наличие аскаридоза (1 пациент), с вероятностью $\mathrm{P}=0,8$ - его отсутствие (4 пациента). При наличии холмистых контуров - наоборот, с вероятностью $\mathrm{P}=0,8$ аскаридоз присутствует (7 пациентов), с вероятностью $\mathrm{P}=0,2$ - отсутствует (2 пациента).

Подобным же образом может быть проанализировано дерево решений с учетом как данных клинической симптоматики, так и УЗИ, представленное на рис. 3. 


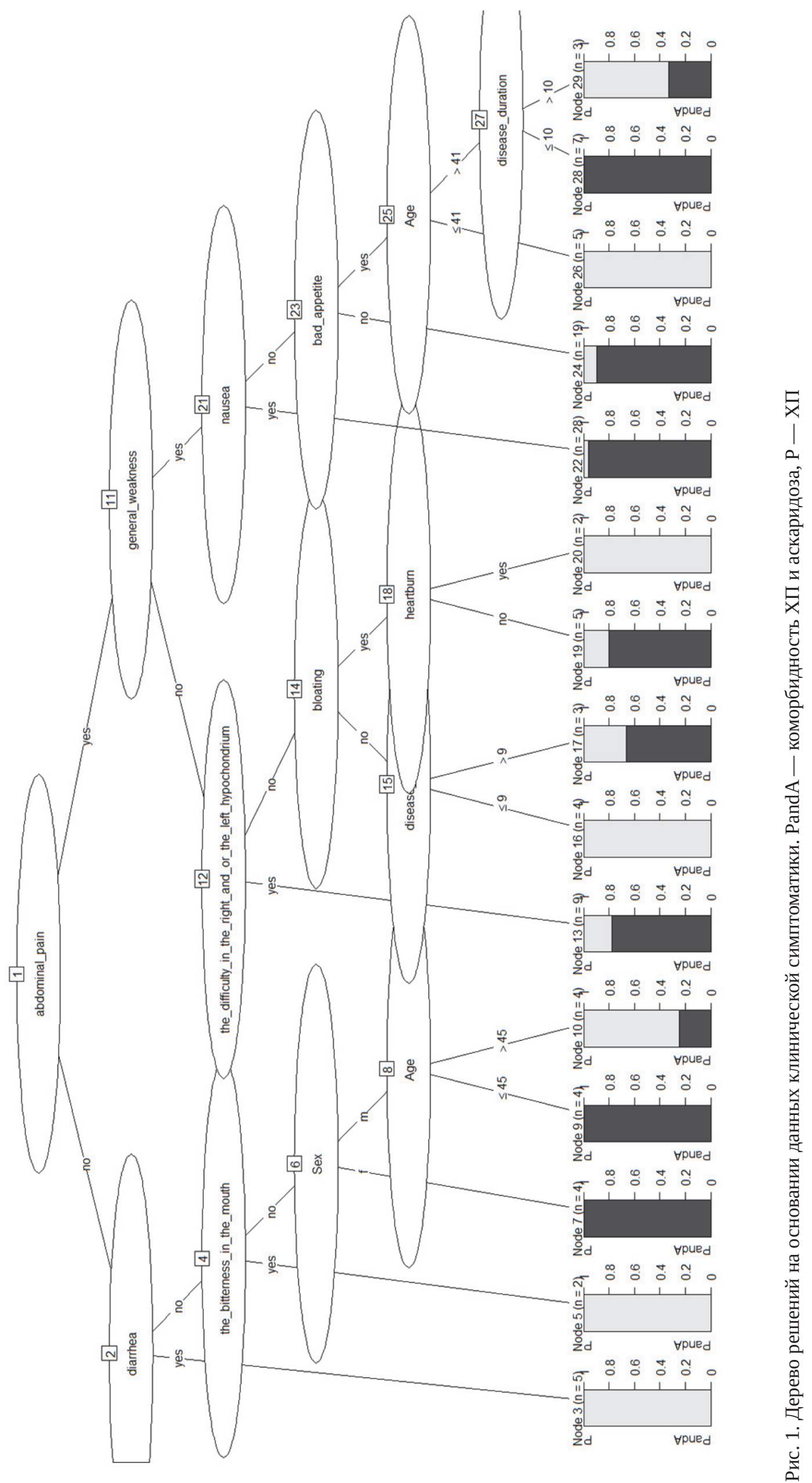




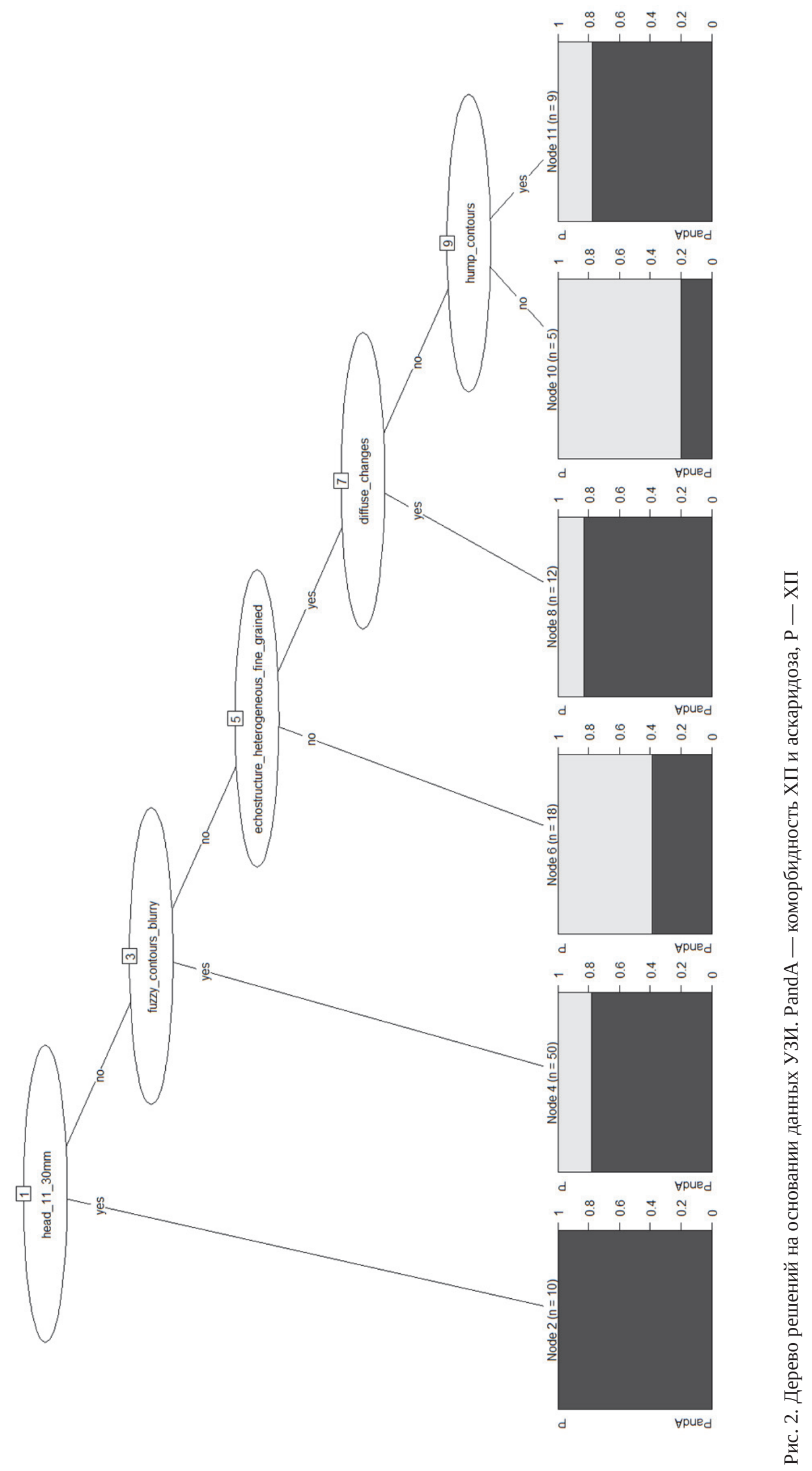




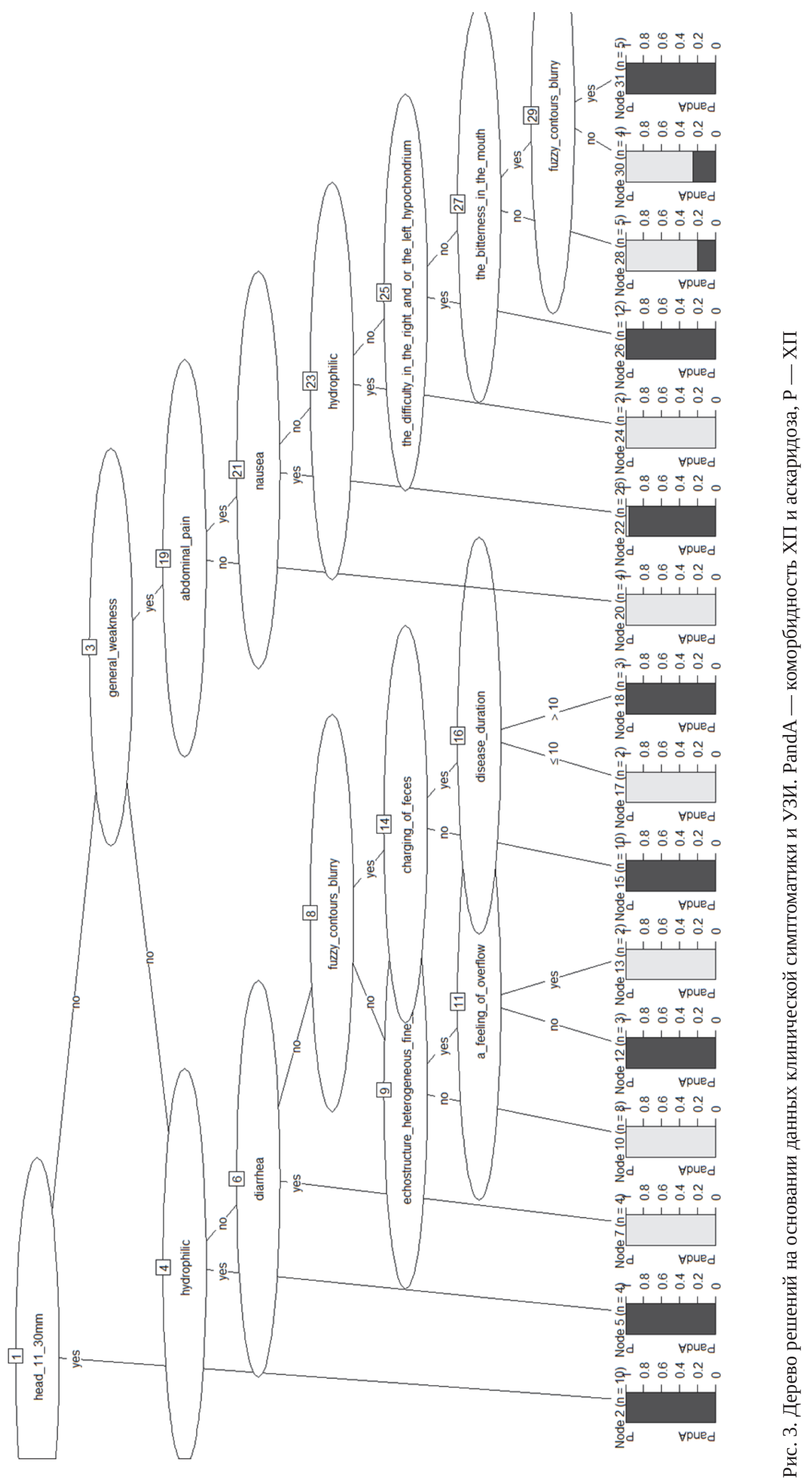


Важно отметить, что с использованием дерева решений получаем частоту использования атрибутов для классификации коморбидных состояний, указанную в табл. 3. В таблице указано использование атрибутов в порядке убывания частоты (\%).

Исследована погрешность диагностирования с использованием дерева (рис. 3). В целом на исследуемых данных погрешность составляет 2,9 \% (т. е. 3 случая из 104). При этом ошибочно диагностируется один случай из 29 при отсутствии аскаридоза и 2 случая из 72 при его наличии.

Таблица 3

\section{Использование атрибутов в дереве решений с учетом данных клинической симптоматики и УЗИ}

\begin{tabular}{|c|c|}
\hline Атрибут & $\begin{array}{c}\text { Использование } \\
\text { в дереве рис. } 3\end{array}$ \\
\hline Увеличение головки (11-30 мм) & $100,0 \%$ \\
\hline Общая слабость & $90,4 \%$ \\
\hline Гидрофильная & $61,5 \%$ \\
\hline Абдоминальная боль & $55,8 \%$ \\
\hline Тошнота & $51,9 \%$ \\
\hline Нечеткие размытые контуры & $35,6 \%$ \\
\hline Диарея & $30,8 \%$ \\
\hline $\begin{array}{c}\text { Тяжесть в правом и/или левом } \\
\text { подреберье (эпигастрии) }\end{array}$ & $25,0 \%$ \\
\hline Чередование запора и диареи & $14,4 \%$ \\
\hline Горечь во рту & $13,5 \%$ \\
\hline $\begin{array}{c}\text { Неоднородная мелкозернистая } \\
\text { эхоструктура }\end{array}$ & $12,5 \%$ \\
\hline Ощущение переполнения & $4,8 \%$ \\
\hline Длительность болезни & $4,8 \%$ \\
\hline
\end{tabular}

Выводы. Проведенное исследование показало, что сопутствующий аскаридоз усложнял ход ХП, увеличивая частоту болевого, диспепсического, энтеропанкреатического синдромов, а также усиливая изменения в ПЖ, выявляемые при проведении УЗИ. Имеющийся или перенесенный в прошлом аскаридоз влиял на клиническое течение ХП, усложняя его, усугубляя ТН - снижение массы тела, анемию, гиповитаминозы, иммунодефицит и другие проявления, которые отображаются на качестве жизни пациента. Выявление аскаридоза позволит повысить точность лечения ХП относительно этиологии, а реабилитационные мероприятия будут успешнее.

Для диагностирования аскаридоза может быть использована методика на основании построения дерева решений. Это одна из составляющих технологии data mining, основанная на алгоритме C5.0. При этом могут быть использованы данные как клинической симптоматики, так и УЗИ. Для каждого из видов исследований может быть построено отдельное дерево. В перспективе дальнейших исследований планируем использовать другие алгоритмы data mining, например, метод последовательного покрытия с построением классификационных правил.

\section{Литература.}

1. Афанасьєв С. В. Місце традиційного ультразвукового дослідження у діагностиці хронічного панкреатиту / С. В. Афанасьєв // Сучасна гастроентерологія. — 2003. — № 1. - С. 72-74.

2. Бабінець Л. С. Аскаридоз і хронічний панкреатит: спільні етіопатогенетичні аспекти, оптимізація лікування в практиці сімейного лікаря / Л. С. Бабінець, Ю.В.Дроняк // Сімейна медицина. — 2014. — № 2. - C. 101-104.

3. Губергріц Н. Б. Практична панкреатологія / Н. Б. Губергріц, С. В. Скопіченко. - Донецьк : Либідь, 2007. -244 c.

4. Клинико-патогенетическая оценка информативности и современные возможности оптимизации ультразвуковой диагностики хронического рецидивирующего панкреатита / Н. Б. Губергриц, Н. Е. Баринова, В. В. Беляев [и др.] // Медицинская визуализация. - 2002. — № 1. — С. 48-58.

5. Сучасні методи дослідження в гастроентерології : метод. посібник / Ю. М. Степанов, М.Б. Щербиніна, I. М. Кононов [та ін.]. - Дніпропетровськ : Свідлер, 2006. - 155 c.

6. Diseases of pancreas / M. W. Buchler, W. Uhl, P. Malfertheiner, M. G. Sarr. — Karger, 2004. — 212 p.

7. Kocher H. M. Chronic pancreatitis / H. M. Kocher // Am. Fam. Physician. - 2008. - Vol. 77, No. 5. P. 661-662.

8. Pancreatic duct ascariasis: sonographic diagnosis a case report / A. Agarwal, V. Chowdhury, N. Srivastava [et al.] // Trop. Gastroenterol. - 2005. - Vol. 26, No. 4. - P. 197-198.

9. Martsenyuk V. P. Qualitative analysis of the antineoplastic immunity system on the basis of a decision tree / V. P. Martsenyuk, I. Y. Andrushchak, I. S. Gvozdetska // Cybern. Syst. Anal. — 2015. - Vol. 51, No. 3. P. 461-470.

10. On multivariate method of qualitative analysis of Hodgkin - Huxley model with decision tree induction / V. Martsenyuk, K. Warwas, K. Augustynek [et al.] // 16th International Conference on Control, Automation and Systems (ICCAS 2016) (16-19 October, 2016, Gyeongju, South Korea). — 2016. — P. 489-494. 
11. Qualitative analysis of compartmental dynamic system using decision-tree induction / A. S. Lyapandra, V. P. Martsenyuk, I. S. Gvozdetska [et al.] // The 8th IEEE International Conference on Intelligent Data Acquisition and Advanced Computing Systems: Technology and Applications (IDAACS’ 2015) (24-26 September, 2015, Warsaw, Poland). — 2015. — P. 688692.

\section{References.}

1. Afanas'ev, S. V. (2003). Mistse traditsiinogo ul'trazvukovogo doslidzhennya u diagnostitsi khronichnogo pankreatitu [Place of traditional ultrasound in the diagnosis of chronic pancreatitis]. Suchasna gastroenterologiya (Modern gastroenterology), 1, 72-74 [in Ukrainian].

2. Babinets', L. S., \& Dronyak, Yu. V. (2014). Askaridoz i khronichnii pankreatit: spil'ni etiopatogenetichni aspekti, optimizatsiya likuvannya v praktitsi simeinogo likarya [Ascaridosis and chronic pancreatitis common etiopathogenetic aspects, optimization of treatment in the practice of the family doctor]. Simeina meditsina (Family medicine), 2, 101-104 [in Ukrainian].

3. Gubergrits, N. B., \& Skopichenko, S. V. (2007). Praktichna pankreatologiya [Practical pancreatology]. Donets'k: Libid' [in Ukrainian].

4. Gubergrits, N. B., Barinova, N. E., Belyaev, V. V., Lukashevich, G. M., Zagorenko, Yu. A., ... Fomenko, P. G. (2002). Kliniko-patogeneticheskaya otsenka informativnosti i sovremennye vozmozhnosti optimizatsii ul'trazvukovoi diagnostiki khronicheskogo retsidiviruyushchego pankreatita [Clinical and pathogenetic assessment of informativeness and modern possibilities of optimization of ultrasonic diagnostics of chronic recurrent pancreatitis]. Meditsinskaya vizualizatsiya (Medical imaging), 1, 48-58 [in Russian].

5. Stepanov, Yu. M., Shcherbinina, M. B., \& Kononov, I. M. (2006). Suchasni metodi doslidzhennya v gastroenterologii [Modern methods of research in gastroenterology]: toolkit. Dnipropetrovs'k: Svidler [in Ukrainian].

6. Buchler, M. W., Uhl, W., Malfertheiner, P., \& Sarr, M. G. (2004). Diseases of pancreas. Karger.

7. Kocher, H. M. (2008). Chronic pancreatitis. Am. Fam. Physician, 77(5), 661-662.

8. Agarwal, A., Chowdhury, V., Srivastava, N., Khera, G., \& Singh, S. (2005). Pancreatic duct ascariasis: sonographic diagnosis - a case report. Trop. Gastroenterol., 26(4), 197-198.

9. Martsenyuk, V. P., Andrushchak, I. Y. \& Gvozdetska, I. S. (2015). Qualitative analysis of the antineoplastic immunity system on the basis of a decision tree. Cybern. Syst. Anal., 51(3), 461.

10. Martsenyuk, V., Warwas, K., Augustynek, K., KlosWitkowska, A., Karpinskyi, V., Klymuk, N., \& Mayhruk, Z. (2017). On multivariate method of qualitative analysis of Hodgkin - Huxley model with decision tree induction. 16th International Conference on Control, Automation and Systems (ICCAS 2016) (16-19 October, 2016, Gyeongju, South Korea) (pp. 489-494). 11. Lyapandra, A. S., Martsenyuk, V. P., Gvozdetska, I. S., Szklarczyk, R., \& Rajba, S. A. (2015). Qualitative analysis of compartmental dynamic system using decision-tree induction. The 8th IEEE International Conference on Intelligent Data Acquisition and Advanced Computing Systems: Technology and Applications (IDAACS’ 2015) (24-26 September, 2015, Warsaw, Poland) (pp. 688-692). 